\title{
First dutch experience of the accurate neo self-expanding supra-annular valve for valve-in-valve transcatheter aortic valve implantation
}

\author{
K. H. Soon ${ }^{1}$ N. H. M. Kooistra' ${ }^{1}$ M. Voskuil' - A. O. Kraaijeveld ${ }^{1}$ P. R. Stella ${ }^{1}$ \\ Published online: 9 February 2018 \\ (c) The Author(s) 2018. This article is an open access publication.
}

We describe the first two cases of valve-in-valve (ViV) transcatheter aortic valve implantation (TAVI) using Accurate Neo device in the Netherlands.

A 73-year-old man underwent transfemoral ViV-TAVI for severe stenosis in his 25-mm Carpentier-Edwards Perimount bioprosthesis. After balloon predilatation, a mediumsize Accurate Neo was advanced to the bioprosthetic annulus. First, its upper part was released with the upper crowns positioned below the superior margin of the pre-existing bioprosthesis (Fig. 1a; [1]). Next, the device was deployed by releasing the lower crowns without ventricular pacing (Movie-1). The results were excellent.

A 79-year-old man underwent transfemoral ViV-TAVI for severe regurgitation of his 27-mm Mosaic bioprosthesis. A small-size Accurate Neo was deployed with its upper crowns positioned along the eyelets of the bioprosthesis (Fig. 1b). After balloon postdilatation, a paravalvular leak was reduced to trace.
Accurate Neo, a self-expanding supra-annular valve, has the advantages of improved ViV haemodynamics and topdown deployment without pacing. However, it is non-repositionable.

Open Access This article is distributed under the terms of the Creative Commons Attribution 4.0 International License (http:// creativecommons.org/licenses/by/4.0/), which permits unrestricted use, distribution, and reproduction in any medium, provided you give appropriate credit to the original author(s) and the source, provide a link to the Creative Commons license, and indicate if changes were made.

\section{Literatur}

1. Bapak V. Valve-in-valve apps: why and how they were developed and how to use them. EuroIntervention. 2014;10:U44-U51.
Electronic supplementary material The online version of this article (https://doi.org/10.1007/s12471-018-1087-3) contains supplementary material, which is available to authorized users.

K. H. Soon

soonkh@yahoo.com

1 Division of Heart \& Lungs, Department of Cardiology, University Medical Center Utrecht, Utrecht, The Netherlands 
Fig. 1 a Positioning of an Accurate Neo device inside a Carpentier-Edwards Perimount aortic bioprosthesis with its upper crowns sitting just below the superior margin of the bioprosthesis (arrows). b Positioning of an Accurate Neo device inside a Mosaic aortic bioprosthesis with its upper crowns positioned along with the eyelets of the bioprosthesis (arrows)
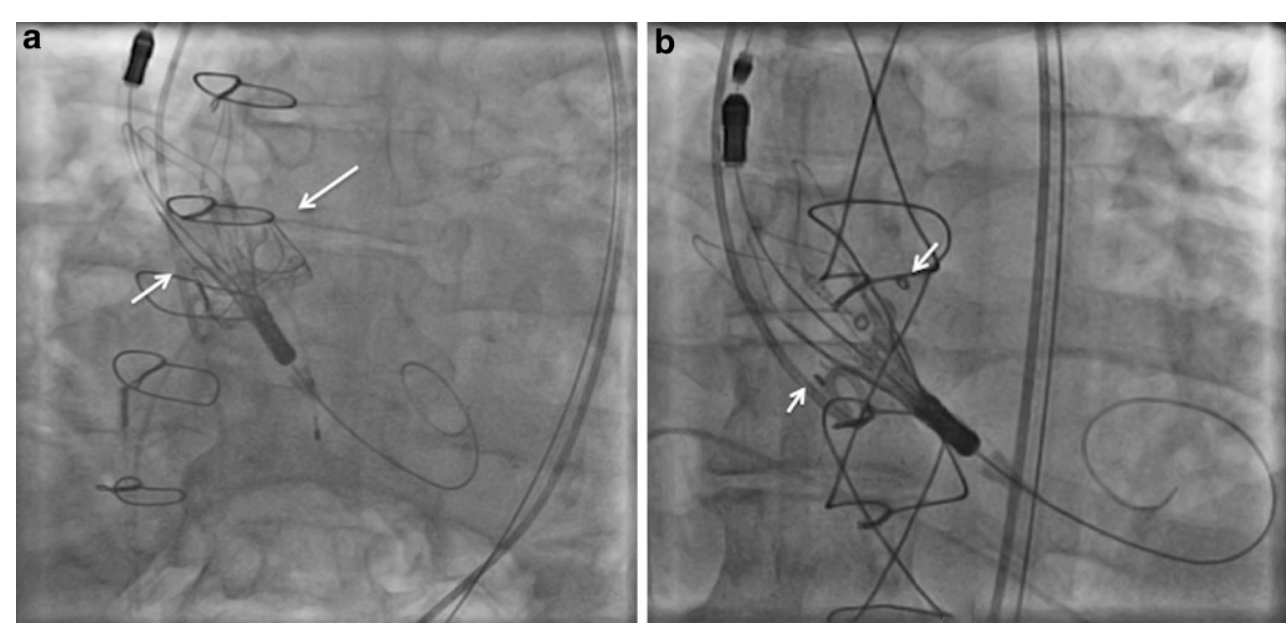